\title{
DTI Correlates of Cognition in Conventional MRI of Normal-Appearing Brain in Patients with Clinical Features of Subacute Combined Degeneration and Biochemically Proven Vitamin $B_{12}$ Deficiency
}

\author{
P.K. Gupta, R.K. Gupta, R.K. Garg, Y. Rai, B. Roy, C.M. Pandey, H.S. Malhotra, and P.A. Narayana
}

\begin{abstract}
BACKGROUND AND PURPOSE: Vitamin $B_{12}$ deficiency may cause neural injury that results in cognitive deficits. The main purpose of our study was to evaluate morphometric and microstructural changes in the brain and relate them to cognition in subacute combined degeneration of the spinal cord and patients with biochemically deficient vitamin $B_{12}$.
\end{abstract}

MATERIALS AND METHODS: Fifty-one patients were recruited and underwent nerve-conduction velocity tests and routine hematologic examinations. Serum vitamin $B_{12}$ and homocystine levels were also measured. All patients and 46 age- and sex-matched controls underwent cervical spine and brain MR imaging along with cognition tests. MR imaging included conventional scans and DTI. Voxel-based morphometry was performed for determining the WM and GM volumes, based on T1-weighted images. DTI measures that included fractional anisotropy, ADC, radial diffusivity, and axial diffusivity were determined by using tract-based statistics.

RESULTS: None of the patients showed any abnormality on conventional MR imaging. No significant changes in GM and WM volumes were observed in patients compared with controls. Significant reductions in the fractional anisotropy and an increase in ADC and radial diffusivity values were observed in multiple brain regions in patients compared with controls. These changes were confirmed on the region-of-interest analysis. Neuropsychological scores were significantly different in patients compared with controls and showed significant correlation with fractional anisotropy and radial diffusivity in a few brain regions.

CONCLUSIONS: Microstructural changes are seen in WM regions on DTI in patients with vitamin $B_{12}$ deficiency and correlate with cognition scores. DTI can be used for objective assessment of microstructural changes in the brain in vitamin $B_{12}$ deficiency.

ABBREVIATIONS: $A D=$ axial diffusivity; $F A=$ fractional anisotropy; $\mathrm{MNI}=$ Montreal Neurological Institute; $\mathrm{RD}=$ radial diffusivity; $\mathrm{SACD}=$ subacute combined degeneration; TBSS $=$ tract-based spatial statistics

B vitamins contribute to CNS development and proper functioning by acting as a cofactor in numerous catalytic reactions in the human body that are required for the synthesis and functioning of neurotransmitters and myelination. A deficiency of vitamin $B_{12}$ may result in injury to the neural tissue. A limited

Received August 19, 2013; accepted after revision September 9.

From the Department of Neurology (P.K.G., R.K.Garg, H.S.M.), King George's Medical University, Lucknow, Uttar Pradesh, India; Department of Radiology and Imaging (R.K.Gupta, B.R.), Fortis Memorial Research Institute, Gurgaon, Haryana, India; Department of Radiodiagnosis (Y.R.) and Biostatistics (C.M.P.), Sanjay Gandhi Postgraduate Institute of Medical Sciences, Lucknow, Uttar Pradesh, India; and Department of Diagnostic and Interventional Imaging (P.A.N.), University of Texas Medical School at Houston, Houston, Texas.

This work was supported by grant BT/IN/German/04/RKG/2010 from the Department of Biotechnology, Ministry of Science and Technology, Government of India Please address correspondence to Rakesh K. Gupta, MD, Department of Radiology and Imaging, Fortis Memorial Research Institute, Gurgaon, Haryana-122002; e-mail: rakeshreel@gmail.com

-- Indicates open access to non-subscribers at www.ajnr.org

Evidence-Based Medicine Level 2.

http://dx.doi.org/10.3174/ajnr.A3785 number of clinical studies in children demonstrated a correlation between vitamin $B_{12}$ deficiency and cognition. ${ }^{1,2}$ Studies in elderly subjects suggest that vitamin $B_{12}$ deficiency is associated with cognitive decline and may contribute to Alzheimer dementia, ${ }^{3,4}$ whereas others have failed to demonstrated an increased risk. ${ }^{5,6}$

Subjects with $\mathrm{B}_{12}$ deficiency may also show changes in the posterolateral column of the spinal cord on MR imaging. Clinical symptoms relating to neuropathy and spinal cord involvement, referred to as subacute combined degeneration (SACD) of the spinal cord, are common in adult subjects with $\mathrm{B}_{12}$ deficiency. Changes in the brain parenchyma on MR imaging have been sporadically reported, with poor sensitivity. ${ }^{7,8}$ In a cross-sectional study on an elderly population, a reduction in brain volume was observed with $\mathrm{B}_{12}$ deficiency. ${ }^{9}$ There are isolated reports of brain demyelination in patients with SACD, which may or may not show resolution following vitamin $\mathrm{B}_{12}$ replacement. ${ }^{10,11}$

Elderly populations with vitamin $\mathrm{B}_{12}$ deficiency are reported to show whole-brain atrophy and white matter damage. ${ }^{12,13} \mathrm{On}$ 
the basis of a number of case reports, brain atrophy is also a pathologic feature in infants with vitamin $\mathrm{B}_{12}$ deficiency. ${ }^{14-16}$ Advanced MR imaging-based modalities such as DTI and MR spectroscopy showed abnormalities in a variety of diffuse neurologic disorders, whereas conventional MR imaging findings appeared normal. We hypothesized that patients with clinical symptoms of SACD and biochemical evidence of vitamin $B_{12}$ deficiency will have an associated cognitive decline and microstructural alterations in brain WM on DTI, even when conventional MR imaging findings appear normal. To verify this hypothesis, we performed whole-brain DTI and cognitive assessment in patients who presented with clinical signs of SACD and a biochemical deficiency of vitamin $\mathrm{B}_{12}$. The DTI measures were correlated with neuropsychological test scores. We also performed voxel-based morphometry analysis for volumetric changes in GM and WM. To the best of our knowledge, this is the first study to quantify the microstructural changes in normal-appearing brain on MR imaging in patients with SACD and biochemically confirmed vitamin $B_{12}$ deficiency.

\section{MATERIALS AND METHODS}

Patients with clinical features of peripheral neuropathy suspected of having spinal cord involvement and clinically labeled as having SACD were included in this study. Fifty-one patients who met the above criteria were recruited ( 38 men, 13 women; mean age, $34.6 \pm 12.2$ years; age range, $18-58$ years). Forty-six age- and sex-matched healthy controls (not on any medication and not known to have any disease) were also included in the study (33 men and 13 women; mean age, $31.1 \pm 8.0$ years; age range, $18-53$ years). No significant differences in age and sex were observed between the patient group and healthy controls.

\section{Clinical Assessment}

Neurologic examinations were performed by a neurologist to assess the severity of impairment in patients with vitamin $B_{12}$ deficiency. The patients had detailed nerve-conduction velocity tests and biochemical analysis for serum vitamin $B_{12}$ and homocysteine levels. The diagnosis of vitamin $\mathrm{B}_{12}$ deficiency was based on low serum vitamin $B_{12}$ levels $(<200 \mathrm{pg} / \mathrm{mL}) .{ }^{17}$ The serum homocysteine level was measured by an enzymatic method. ${ }^{18}$ Routine hematologic examinations that included hemoglobin levels and red cell mean corpuscular volume were also performed. Healthy controls were evaluated for serum vitamin $\mathrm{B}_{12}$ levels and neurologic and cognitive status; however, they did not undergo nerveconduction velocity tests and other biochemical analyses. All patients who showed vitamin $B_{12}$ deficiency and had clinical features of SACD underwent MR imaging of both the cervical spine and brain. All patients with normal brain MR imaging findings with or without imaging changes in the cervical spine underwent cognitive testing.

Neuropsychological tests were performed on both patients and controls by an experienced neuropsychologist. This battery included the Trail-Making test, number connection tests A and B, and figure connection tests $\mathrm{A}$ and $\mathrm{B}$ as well as the performance subset of the modified Wechsler Adult Intelligence Scale (modified for the population), which included picture completion, digit symbol, block design, picture arrangement, and object assembly. These tests evaluate visuospatial capacity and visuomotor speed.
The Trail-Making test assesses the visual motor coordination, concentration, attention, mental speed, and memory alteration. ${ }^{19}$ In the number connection and figure connection tests A and $\mathrm{B}$, lower scores represent better performance, whereas in the Wechsler Adult Intelligence Scale, a higher score represents a better performance.

This study protocol was approved by the Institutional Ethics Committee. Informed written consent was obtained from each subject.

\section{MR Imaging}

All MR imaging studies were performed on a 3T MR imaging scanner (Signa Hdxt; GE Healthcare, Milwaukee, Wisconsin). An 8-channel head coil was used for brain MR imaging. T2-weighted axial images were acquired with $\mathrm{TR}=9200 \mathrm{~ms}, \mathrm{TE}=72 \mathrm{~ms}$, $\mathrm{NEX}=1$, section thickness $=3 \mathrm{~mm}$, flip angle $=90^{\circ}$, acquisition matrix $=512 \times 256, \mathrm{FOV}=240 \mathrm{~mm}$, reconstructed matrix $=$ $1024 \times 1024$. Parameters of FLAIR imaging were TR $=9000 \mathrm{~ms}$, $\mathrm{TE}=128 \mathrm{~ms}, \mathrm{TI}=2400 \mathrm{~ms}, \mathrm{NEX}=1$, section thickness $=3 \mathrm{~mm}$, flip angle $=90^{\circ}$, acquisition matrix $=320 \times 256, F O V=240 \mathrm{~mm}$, reconstructed matrix $=512 \times 512$. 3D T1-weighted inversionrecovery-prepared fast-spoiled gradient-echo imaging was performed by using the following parameters: $\mathrm{TR}=8.4 \mathrm{~ms}, \mathrm{TE}=3.3$ $\mathrm{ms}$, number of sections $=184$, section thickness $=1 \mathrm{~mm}$, intersection gap $=0, \mathrm{FOV}=240 \mathrm{~mm}$, image matrix $=512 \times 512$, $\mathrm{NEX}=1$, TI $=400 \mathrm{~ms}$, and flip angle $=13^{\circ}$. DTI data were acquired by using dual spin-echo single-shot echo-planar sequences with 30 uniformly distributed directions with ramp sampling. The acquisition parameters were the following: $\mathrm{TR}=17$ $\mathrm{sec}, \mathrm{TE}=88.7 \mathrm{~ms}$, number of sections $=62$, section thickness $=$ $3 \mathrm{~mm}$, intersection gap $=0, \mathrm{FOV}=240 \times 240 \mathrm{~mm}$, image matrix $=256 \times 256, \operatorname{NEX}=1$, diffusion-weighting $\mathrm{b}$ factor $=1000$ $\mathrm{s} / \mathrm{mm}^{2}$.

MR imaging of the cervical spine was performed on a 12channel head-neck-spine coil by using T1 FLAIR and T2 fast recovery FSE in the sagittal and axial planes. Imaging parameters for T1 FLAIR were the following: TR $=2496 \mathrm{~ms}$, TE $=25$ $\mathrm{ms}$, TI $=1013 \mathrm{~ms}, \mathrm{NEX}=1$, section thickness $=3 \mathrm{~mm}$, flip angle $=90^{\circ}$, acquisition matrix $=384 \times 256$, FOV $=259.99$ $\mathrm{mm}$, reconstructed matrix $=512 \times 512$. T2 fast recovery FSE in the sagittal plane had the following parameters: $\mathrm{TR}=2080$ $\mathrm{ms}, \mathrm{TE}=87.17 \mathrm{~ms}, \mathrm{NEX}=2$, section thickness $=3 \mathrm{~mm}$, flip angle $=90^{\circ}$, acquisition matrix $=384 \times 256, \mathrm{FOV}=260 \mathrm{~mm}$, reconstructed matrix $=512 \times 512$. Imaging parameters for $\mathrm{T} 2$ fast recovery FSE in the axial plane were the following: $\mathrm{TR}=$ $3120 \mathrm{~ms}, \mathrm{TE}=124 \mathrm{~ms}, \mathrm{NEX}=2$, section thickness $=3 \mathrm{~mm}$, flip angle $=90^{\circ}$, acquisition matrix $=320 \times 224, \mathrm{FOV}=180$ $\mathrm{mm}$, reconstructed matrix $=512 \times 512$.

\section{MR Imaging Analysis}

Structural Analysis. Voxel-based morphometry analysis was performed by using SPM8 (Wellcome Department of Imaging Neuroscience, London, UK; http://www.fil.ion.ucl.ac.uk/spm) to determine possible changes in the GM and WM volumes. Before processing, the images were visually inspected for possible artifacts. For tissue segmentation, a customized template was built from the sample by using a nonlinear registration 
algorithm (DARTEL toolbox in SPM8). ${ }^{20}$ This template was registered to the Montreal Neurological Institute (MNI) template for group comparisons. The Jacobian determinants from the normalization procedure were used to modulate the voxelbased morphometry data to preserve the WM and GM volumes. Individual GM and WM images were smoothed with an isotropic Gaussian kernel of 6-mm full width at half maximum before statistical analysis. Global volumes of GM and WM were assessed from segmented images by using the VBM8 toolbox in SPM8 after correcting for age and sex.

\begin{tabular}{|c|c|c|c|}
\hline Test & Subject & Mean & $P$ Value \\
\hline \multirow[t]{2}{*}{ Digit symbol } & Control & $11.19 \pm 2.04$ & $<.001$ \\
\hline & Patient & $7.22 \pm 2.79$ & \\
\hline \multirow[t]{2}{*}{ Number connection test $A$} & Control & $39.63 \pm 9.4$ & $<.001$ \\
\hline & Patient & $61.67 \pm 25.09$ & \\
\hline \multirow[t]{2}{*}{ Number connection test B } & Control & $64.59 \pm 16.61$ & $<.001$ \\
\hline & Patient & $101.59 \pm 43.37$ & \\
\hline \multirow[t]{2}{*}{ Picture completion } & Control & $14.89 \pm 1.23$ & $<.001$ \\
\hline & Patient & $12.04 \pm 1.89$ & \\
\hline \multirow[t]{2}{*}{ Block designing } & Control & $12.74 \pm 1.1$ & $<.001$ \\
\hline & Patient & $9.08 \pm 2.31$ & \\
\hline \multirow[t]{2}{*}{ Picture arrangement } & Control & $13.35 \pm 11.53$ & $<.001$ \\
\hline & Patient & $11.53 \pm 2.22$ & \\
\hline \multirow[t]{2}{*}{ Object assembling } & Control & $11.19 \pm 1.26$ & $<.001$ \\
\hline & Patient & $9.88 \pm 1.90$ & \\
\hline \multirow[t]{2}{*}{ Figure connection test $\mathrm{A}$} & Control & $56.37 \pm 21.72$ & $<.001$ \\
\hline & Patient & $80.49 \pm 41.64$ & \\
\hline \multirow{2}{*}{ Figure connection test $\mathrm{B}$} & Control & $77.85 \pm 23.92$ & $<.001$ \\
\hline & Patient & $125.61 \pm 40.99$ & \\
\hline
\end{tabular}

\section{Diffusion Tensor Image Processing}

The Diffusion Toolbox software tool in the FMRIB Software Library (FSL; http://www.fmrib.ox.ac.uk/fsl/fdt/index.html) was used for calculating the DTI indices fractional anisotropy (FA), ADC, axial diffusivity (AD), and radial diffusivity (RD). The DWI was corrected for eddy current-induced distortions and minor head movements by using affine registration to the reference $\mathrm{B} 0$ images. The Brain Extraction Tool was used for extracting the brain. ${ }^{21}$

\section{Tract-Based Spatial Statistics and Voxelwise Analysis}

Voxelwise analysis of FA was performed by using tract-based spatial statistics (TBSS), ${ }^{22}$ part of the FSL package. ${ }^{23}$ Individual skeletonized FA maps were aligned to the MNI 152 template by using the Nonlinear Registration Tool in FMRIB. ${ }^{24}$ Each subject's aligned FA map was then projected onto this skeleton, and the voxelwise general linear model was applied by using permutationbased nonparametric testing, corrected for multiple comparisons. Using the same registration parameters from the FA maps, we also spatially transformed $\mathrm{ADC}, \mathrm{RD}$, and $\mathrm{AD}$ maps to the MNI space.

\section{Region-of-Interest Analysis}

In addition to the TBSS analysis, the region-of-interest analysis was also performed on those regions that were observed to be significantly different on the TBSS analysis. Elliptic regions of interest of sizes varying from 25 to $50 \mathrm{~mm}^{2}$ were placed on different regions in the FA maps of patients and controls.

\section{Statistical Analysis}

The differences in the neuropsychological scores between patients and controls were analyzed with independent $t$ tests by using the

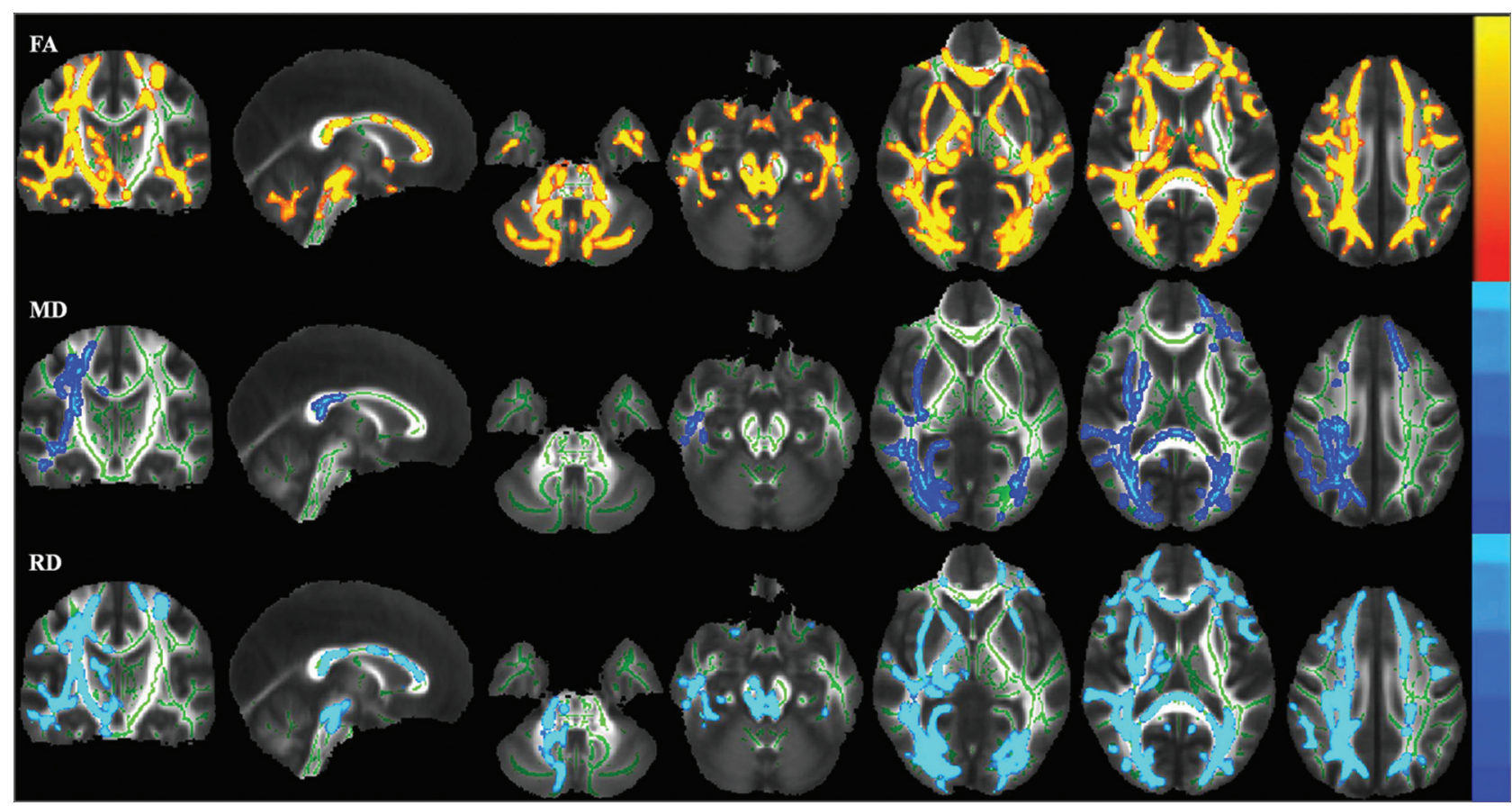

FIG 1. Differences in DTI measures between patients and controls. Statistical maps show voxels that exhibit differences in DTI parameters in patients versus controls (red and yellow colors, according to the lower and higher significance, respectively; dark blue and light blue colors, according to the lower and higher significance, respectively). FA is significantly decreased, and ADC and RD are increased in the patient group compared with controls. Differences are widespread and evident in various WM regions. All WM tracts are overlaid on a 1-mm standard image in MNI 152 (TBSS analysis, 2-sample, $P<.05$, threshold-free cluster enhancement corrected). 


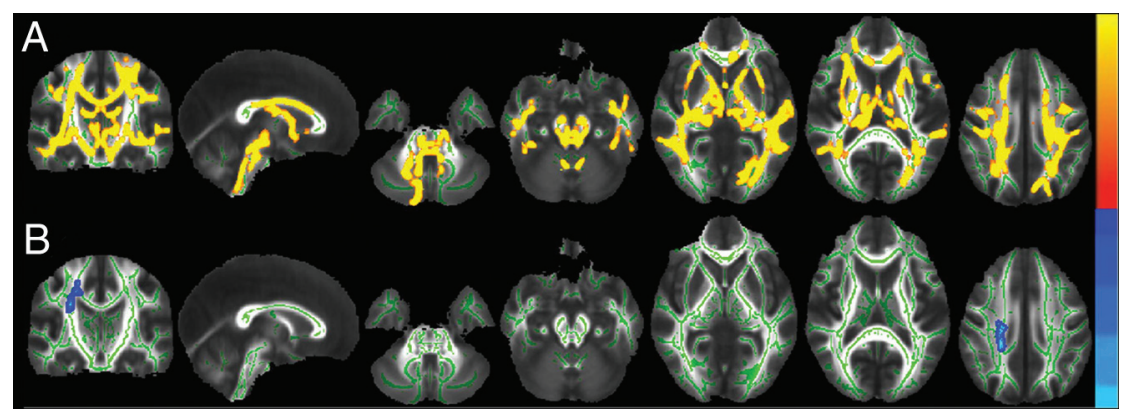

FIG 2. Correlation between FA and neuropsychological scores. The statistical map shows voxels that correlate with neuropsychological scores. A, FA positively correlates with Digit Symbol score in various WM regions. $B$, Negative correlation between FA and the number connection test score was observed. All WM tracts are overlaid on a 1-mm standard image in MNI 152 (TBSS analysis, $P<.05$, threshold-free cluster enhancement corrected).

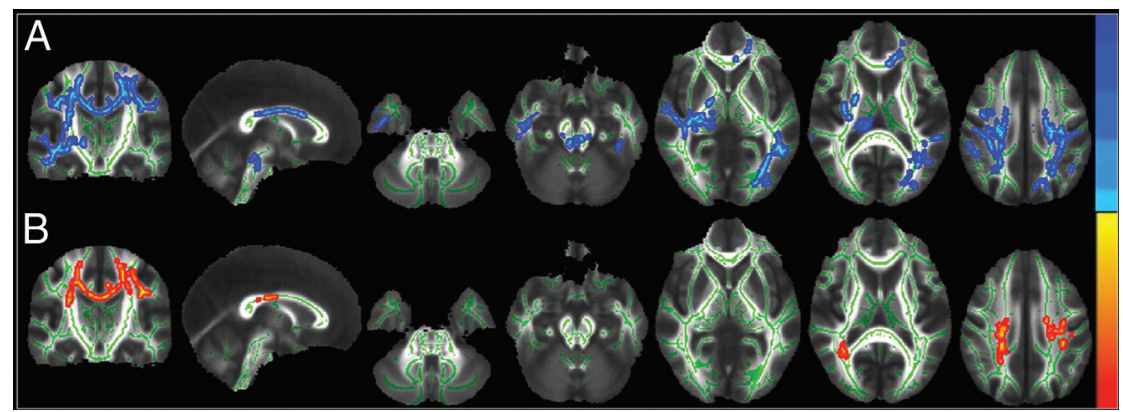

FIG 3. Correlation between RD and neuropsychological scores. Statistical map shows voxels that correlate with neuropsychological scores. A, RD negatively correlates with the Digit Symbol score in various WM regions. $B, A$ positive correlation between $\mathrm{RD}$ and the number connection test score was observed. All WM tracts are overlaid on a 1-mm standard image in MNI 152 (TBSS analysis, $P<.05$, threshold-free cluster enhancement corrected).

Statistical Package for Social Sciences software, Version 16.01 (IBM, Armonk, New York). The correlation between the neuropsychological score and DTI-derived indices of WM was based on the Pearson coefficient. All statistical analyses were based on a 2-tailed test with an $\alpha$ level of $<.05$ for statistical significance.

Morphologic differences between the patients and control subjects were estimated by using an independent-samples $t$ test at the voxel level within the general linear model framework of statistical parametric mapping. Comparison between patients and control subjects was made for 2 different contrasts, corresponding to an increase (patient $>$ controls) or decrease (patient $<$ controls) in GM and WM volumes. A false discovery rate at $P<.05$ was used. Differences were considered significant if the cluster size was $>15 .^{25}$

The general linear model was applied across all subjects to identify the brain regions in which the patient group showed significant differences in $\mathrm{FA}, \mathrm{ADC}, \mathrm{AD}$, and $\mathrm{RD}$ relative to the healthy control group. The correlation analyses were performed to study the relationship between neuropsychological scores and each of the DTI-derived indices FA, ADC, $\mathrm{AD}$, and RD in the WM by using neuropsychological scores as regressors in the framework of a general linear model. The effects of age and sex were regressed out in these models. DTI-derived maps were included in a nonparametric permutation-based group model by using "Randomize" in FSL. An independent $t$ test was used to determine the differences in $\mathrm{FA}, \mathrm{ADC}, \mathrm{RD}$, and $\mathrm{AD}$ values obtained from the region-of-interest analysis between patient and control groups.

\section{RESULTS}

\section{Clinical Assessment}

The mean hemoglobin level in patients with vitamin $\mathrm{B}_{12}$ deficiency was $10.95 \pm$ $2.28 \mathrm{~g} / \mathrm{dL}$ (range, $6.8-16 \mathrm{~g} / \mathrm{dL}$ ), the mean serum vitamin $B_{12}$ concentration was $145.26 \pm 42.07 \mathrm{pg} / \mathrm{mL}$ (range, $26-199 \mathrm{pg} /$ $\mathrm{mL})$, the mean cell volume was $103.6 \pm$ $12.26 \mathrm{fL}$ (range, 81.3-122.3 fL), and the serum homocysteine level was $19.84 \pm$ $7.32 \mu \mathrm{mol} / \mathrm{L}$ (range, $7.9-41.29 \mu \mathrm{mol} / \mathrm{L}$ ). The mean serum vitamin $B_{12}$ concentration in healthy controls was $330.22 \pm$ $135.22 \mathrm{pg} / \mathrm{mL}$ (range, 255-678 pg/mL). All patients had gait disturbance, sensory disturbance, mental impairment, and neuropathy. A total of $11.8 \%$ of patients presented clinically with pyramidal tract damage. Differences were observed in all the neuropsychological scores between patients and controls (Table).

\section{Conventional MR Imaging}

MR imaging of the cervical spine showed T2 hyperintensity in the posterior spinal cord in 7 patients and diffuse hyperintensity of the cervical cord in 1 patient. The remaining 43 patients did not show any abnormality in the cervical cord on conventional MR imaging.

\section{Voxel-Based Morphometry Analysis}

The voxel-based morphometry analysis of the 3D-T1WI did not show a significant difference in the cerebral GM and WM volumes between patients and controls.

\section{TBSS Analysis}

TBSS analysis showed significantly reduced FA values in patients compared with controls in a number of WM regions, which included the frontal, parietal, and temporal lobes and the entire corpus callosum and its associated fibers (Fig 1). The patient group also had a widespread increase in ADC and RD values, predominantly in the right hemisphere tracts (right $>$ left) and the corpus callosum.

\section{Correlation Analysis of DTI Indices with Neuropsychological Scores}

Correlation maps showed a significant positive correlation between the Digit Symbol scores and FA values (Fig 2A) and a significant negative correlation between the number connection test and FA values (Fig $2 B$ ) in many WM bundles ( $P$ corrected $<.05$ ). $\mathrm{RD}$ values were found to correlate negatively with Digit Symbol scores (Fig $3 A$ ) and positively with number connection test scores (Fig 3B). ADC and AD did not show any significant correlation 
with neuropsychological scores. The mean FA and RD values were extracted subject by subject from correlation maps from regions showing significant correlation between DTI indices and neuropsychological scores. Thereafter, Pearson correlation coefficients were obtained by using SPSS in the Digit Symbol test, with FA ( $r=$ $0.63, P<.001$ in patients; $r=0.56, P<.001$ controls $)$ and $\mathrm{RD}$ ( $r=-0.54, P<.001$ in patients; $r=-0.53, P<.001$ in controls $)$; and the number connection test with FA $(r=-0.56, P<.001$ in patients; $r=-0.22, P=.14$ in controls $)$ and RD $(r=0.57, P<$ .001 in patients; $r=0.403, P<.005$ in controls) to verify the results obtained on correlation maps.

\section{Region-of-Interest Analysis}

Region of interest-based analysis confirmed the TBSS results. The results are summarized in Fig 4. AD values did not show a significant change between patients and controls either on the TBSS or region-of-interest analysis.

\section{DISCUSSION}

This study shows widespread changes in the cerebral WM in patients with vitamin $B_{12}$ deficiency in all the DTI metrics, except $\mathrm{AD}$, indicating altered $\mathrm{WM}$ microstructure in multiple regions in these patients. These results were confirmed in the region-of-interest analysis. In addition, all these patients showed significant cognitive decline over the controls. The cognitive scores correlated with DTI measures in various brain regions. Our findings suggest that the microstructure changes in WM are quite widespread in the brain, even when patients showed clinical symptoms related to the spinal cord. We did not observe any volume changes in WM and GM on voxel-based morphometry analysis, suggesting that atrophy is not a major pathologic component in these patients.

FA is widely considered a robust measure of WM organization, and a number of studies have reported abnormalities in FA across both psychotic and affective illnesses. ${ }^{26}$ Disruptions in WM organization (reflected in reductions in FA) can result from various mechanisms, including demyelination as well as axonal loss and lacks pathologic specificity. However, the other DTI-derived measures, such as RD and $\mathrm{AD}$, are thought to reflect myelin and axonal integrity, respectively. ${ }^{27}$ While still controversial, some published literature suggests that $\mathrm{RD}$ is a more sensitive measure of myelin integrity, while $\mathrm{AD}$ reflects axonal integrity. ${ }^{28,29}$ In the current study, we observed increased RD and unchanged $\mathrm{AD}$, suggesting that demyelination, not axonal loss, is the major pathologic substrate in $\mathrm{B}_{12}$ deficiency. Scalabrino and Veber ${ }^{30}$ have demonstrated, in the rat model, that vitamin $B_{12}$ deficiency damages myelin and causes myelin vacuolation and reactive astrocytosis in the CNS. In a recent study, Minn et al ${ }^{31}$ reported that patients with SACD have progressive degeneration in the following sequential order: lower spinal cord, cervical spinal cord, peripheral nerve/optic nerve, and, finally, the brain. Our data do not support this view and show that $\mathrm{B}_{12}$ deficiency causes demyelination in the brain even when it may manifest clinically with SACD.

In a prospective study on 50 patients with vitamin $B_{12}$ deficiency, Reynolds ${ }^{32}$ has shown cognitive impairment or an affective disorder in one-third of patients. Minn et $\mathrm{al}^{31}$ did not find any symptoms of dementia in any of their patients who presented with SACD. However, in the current study, all 51 patients showed sig-
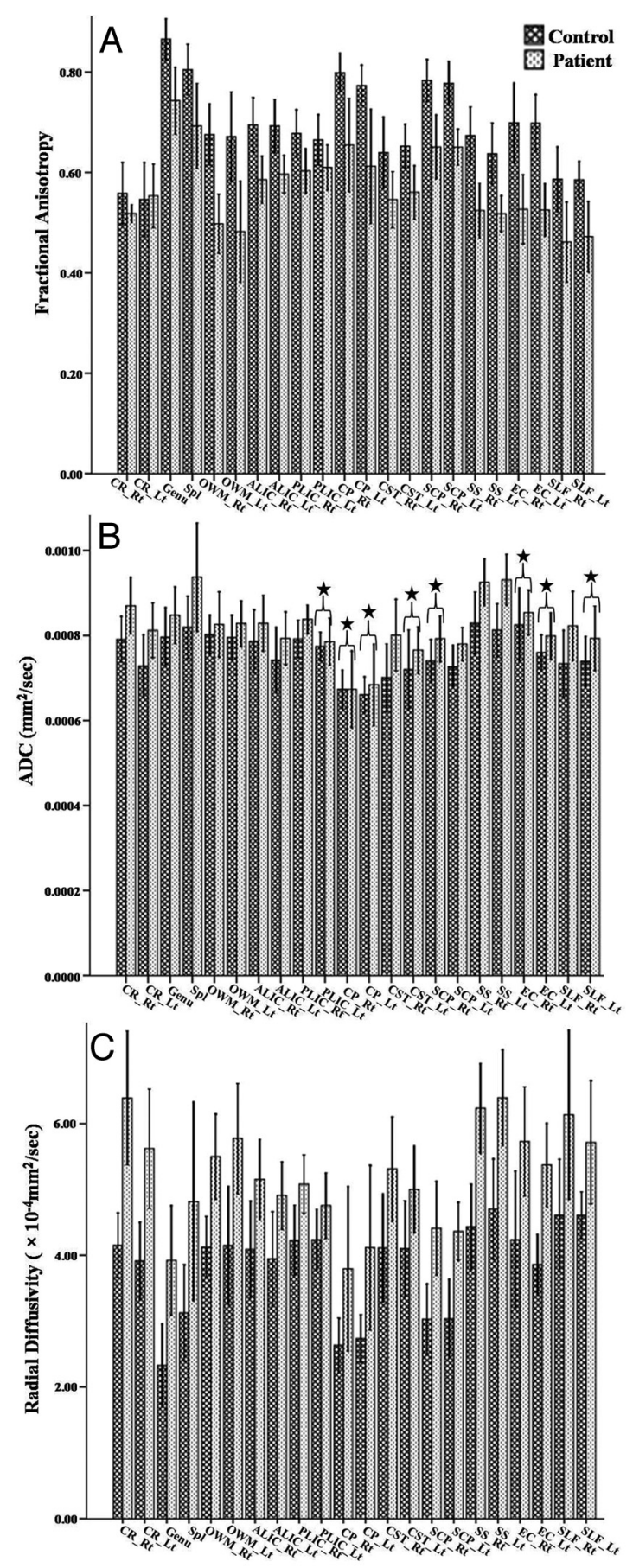

FIG 4. Bar diagram showing significant differences in various white matter regions on DTI metrics in patients compared with controls on region-of-interest analysis. Bars show a significant difference in FA $(A)$, ADC (the asterisk indicates regions with nonsignificant differences) $(B)$, and RD $(C)$ in the specified regions. CR_Rt \& CR_Lt indicates corona radiata right and left; Spl, splenium; OWM_Rt \& OWM_Lt, occipital white matter right and left; ALIC_Rt \& ALIC_Lt, anterior limb of the internal capsule right and left; PLIC_Rt \& PLIC_Lt, posterior limb of the internal capsule right and left; $\overline{C P}_{-}$Rt \& CP_Lt, cerebellar peduncle right and left; CST_Rt \& CST_Lt, corticospinal tract right and left; SCP_Rt \& SCP_Lt, superior cerébellar peduncle right and left; SS_Rt \& SS_Lt, sagittal stratum right and left; EC_Rt \& EC_Lt, external capsule right and left; SLF_Rt \& SLF_Lt, superior longitudinal fasciculus right and left. 
nificant cognitive deficits on neuropsychological tests compared with the age- and sex-matched controls, suggesting that a complete battery of tests may be needed to detect cognitive impairment in these patients. Dementia and cognitive changes have been typically reported in elderly subjects with $B_{12}$ deficiency. In contrast, we detected cognitive deficits in our relatively young patient cohort.

Cognitive decline has been observed at all age groups in patients with vitamin $B_{12}$ deficiency. Sensitive neuropsychological tests have shown an association with vitamin deficiency. ${ }^{33}$ In the current study, we also observed a decline in visuospatial and performance skills in these patients. Another group has reported cognitive decline in patients between 35 and 50 years of age with vitamin $B_{12}$ deficiency, which improved following its supplementation. ${ }^{34}$

Most studies in the geriatric population show conflicting results. Some authors have found a strong causal relationship between cognitive decline and vitamin $\mathrm{B}_{12}$ deficiency, while others have described the relationship as mere coincidence in the geriatric population. ${ }^{3,5,12}$ The mechanism for cognition decline is the accumulation of methylmalonic acid secondary to nonactivation of methylmalonyl-CoA mutase, which is myelinotoxic. ${ }^{35} \mathrm{We}$ also observed a strong correlation of the neuropsychological scores with $\mathrm{FA}, \mathrm{ADC}$, and $\mathrm{RD}$ changes in some brain regions on DTI.

\section{CONCLUSIONS}

Microstructural changes in various brain regions are demonstrated on DTI metrics in patients with SACD, and these are associated with abnormal neuropsychological scores and show a correlation with various specific brain regions. The imaging technique may be of value in the objective assessment of the brain changes in $\mathrm{B}_{12}$ deficiency.

Disclosures: Ponnada Narayana—UNRELATED: Grants/Grants Pending: National Institutes of Health, ${ }^{*}$ Department of Defense. ${ }^{\star \star}$ Money paid to the institution.

\section{REFERENCES}

1. Strand TA, Taneja S, Ueland PM, et al. Cobalamin and folate status predicts mental development scores in North Indian children 12-18 mo of age. Am J Clin Nutr 2013;97:310-17

2. Black MM. Effects of vitamin B12 and folate deficiency on brain development in children. Food Nutr Bull 2008;29:S126-31

3. Clarke R, Birks J, Nexo E, et al. Low vitamin B-12 status and risk of cognitive decline in older adults. Am J Clin Nutr 2007;86:1384-91

4. Kivipelto M, Annerbo S, Hultdin J, et al. Homocysteine and holotranscobalamin and the risk of dementia and Alzheimers disease: a prospective study. Eur J Neurol 2009;16:808-13

5. Lachner C, Steinle NI, Regenold WT. The neuropsychiatry of vitamin B12 deficiency in elderly patients. J Neuropsychiatry Clin Neurosci 2012;24:5-15

6. Ford AH, Flicker L, Thomas J, et al. Vitamins B12, B6, and folic acid for onset of depressive symptoms in older men: results from a 2-year, placebo-controlled, randomized trial. J Clin Psychiatry 2008:69:1203-09

7. Crawford JR, Say D. Vitamin B12 deficiency presenting as acute ataxia. BMJ Case Rep 2013;pii

8. Gürsoy AE, Kolukısa M, Babacan-Yıldız G, et al. Subacute combined degeneration of the spinal cord due to different etiologies and improvement of MRI findings. Case Rep Neurol Med 2013;2013:159649

9. Tangney CC, Aggarwal NT, Li H, et al. Vitamin B12, cognition, and brain MRI measures. Neurology 2011;77:1276-82

10. Silva MT, Cavalcanti JL, Moreira DM. Neuroradiological features of the brain in subacute combined spinal cord degeneration: case report [in Portuguese]. Arq Neuropsiquiatr 2000;58:752-55

11. Katsaros VK, Glocker FX, Hemmer B, et al. MRI of spinal cord and brain lesions in subacute combined degeneration. Neuroradiology 1998;40:716-19

12. Smith $\mathrm{AD}$, Refsum $\mathrm{H}$. Vitamin B-12 and cognition in the elderly. Am J Clin Nutr 2009;89:707S-11S

13. Vogiatzoglou A, Refsum H, Johnston C, et al. Vitamin B12 status and rate of brain volume loss in community-dwelling elderly. Neurology 2008;71:826-32

14. Biancheri R, Cerone R, Schiaffino MC, et al. Cobalamin (Cbl) C/D deficiency: clinical, neurophysiological and neuroradiologic findings in $\mathbf{1 4}$ cases. Neuropediatrics 2001;32:14-22

15. Lövblad K, Ramelli G, Remonda L, et al. Retardation of myelination due to dietary vitamin B12 deficiency: cranial MRI findings. Pediatr Radiol 1997;27:155-58

16. Taskesen M, Yaramis A, Pirinccioglu AG, et al. Cranial magnetic resonance imaging findings of nutritional vitamin B12 deficiency in 15 hypotonic infants. Eur J Paediatr Neurol 2012;16:266-70

17. Remacha AF, Souto JC, Piñana JL, et al. Vitamin B12 deficiency, hyperhomocysteinemia and thrombosis: a case and control study. Int J Hematol 2011;93:458-64

18. Clarke R, Refsum H, Birks J, et al. Screening for vitamin B-12 and folate deficiency in older persons. Am J Clin Nutr 2003;77:1241-47

19. Dhiman RK, Saraswat VA, Verma M, et al. Figure connection test: a universal test for assessment of mental state. J Gastroenterol Hepatol 1995;10:14-23

20. Ashburner J. A fast diffeomorphic image registration algorithm. Neuroimage 2007:38:95-113

21. Smith SM. Fast robust automated brain extraction. Hum Brain Mapp 2002;17:143-55

22. Smith SM, Jenkinson M, Johansen-Berg H, et al. Tract-based spatial statistics: voxelwise analysis of multi-subject diffusion data. Neuroimage 2006;31:1487-505

23. Smith SM, Jenkinson M, Woolrich MW, et al. Advances in functional and structural MR image analysis and implementation as FSL. Neuroimage 2004;23:S208-19

24. Andersson JL, Jenkinson M, Smith S. Non-linear optimisation. FMRIB Analysis Group Technical Reports. TR07JA1. www.fmrib.ox. ac.uk/analysis/techrep. Accessed 2007

25. Margariti PN, Astrakas LG, Tsouli SG, et al. Investigation of unmedicated early onset restless legs syndrome by voxel-based morphometry, T2 relaxometry, and functional MR imaging during the nighttime hours. AJNR Am J Neuroradiol 2012;33:667-72

26. Domen PA, Michielse S, Gronenschild E, et al. Microstructural white matter alterations in psychotic disorder: a family-based diffusion tensor imaging study. Schizophr Res 2013;146:291-300

27. Inano $\mathrm{S}, \mathrm{Takao} \mathrm{H}, \mathrm{Hayashi} \mathrm{N}$, et al. Effects of age and gender on white matter integrity. AJNR Am J Neuroradiol 2011;32:2103-09

28. Song SK, Sun SW, Ramsbottom MJ, et al. Dysmyelination revealed through MRI as increased radial (but unchanged axial) diffusion of water. Neuroimage 2002;17:1429-36

29. Bockhorst KH, Narayana PA, Dulin J, et al. Normobaric hyperoximia increases hypoxia-induced cerebral injury: DTI study in rats. J Neurosci Res 2010;88:1146-56

30. Scalabrino G, Veber D. Normal prions as a new target of cobalamin (vitamin B12) in rat central nervous system. Clin Chem Lab Med 2013;51:601-06

31. Minn YK, Kim SM, Kim SH, et al. Sequential involvement of the nervous system in subacute combined degeneration. Yonsei Med J 2012;53:276-78

32. Reynolds E. Vitamin B12, folic acid, and the nervous system. Lancet Neurol 2006;5:949-60

33. Morris MS. The role of $\mathbf{B}$ vitamins in preventing and treating cognitive impairment and decline. Adv Nutr 2012;3:801-12

34. Aaron S, Kumar S, Vijayan J, et al. Clinical and laboratory features and response to treatment in patients presenting with vitamin B12 deficiency-related neurological syndromes. Neurol India 2005;53:55-58

35. Selhub J, Morris MS, Jacques PF, et al. Folate-vitamin B-12 interaction in relation to cognitive impairment, anemia, and biochemical indicators of vitamin B-12 deficiency. Am J Clin Nutr 2009;89:702S-06S 